\title{
Correction to: On the Signed Complete Graphs with Maximum Index
}

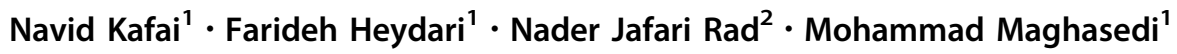

Published online: 23 September 2021

(c) Shiraz University 2021

\section{Correction to: Iran J Sci Technol Trans Sci https://doi.org/10.1007/s40995-021-01199-w}

In this article all degrees are incorrect and appears by an o notation, it is represented by $\mathrm{O} \_\mathrm{G}(\mathrm{v})$ in Introduction section (Page 1, left column, line 3) and Page 5, left column, lines $34,35,36,37,38$.

It should have been

Deg $G(v)$ in the introduction section
In Page 5 lines 34, 35,36,37,38.

$\operatorname{deg} U(v 1)=2$ or $\operatorname{deg} U(v 3)=2$

$\operatorname{deg} U(v 2)=2$ or $\operatorname{deg} U(v 4)=2$

$\operatorname{deg} U(v 3)=\operatorname{deg} U(v 4)=2$

The original article has been corrected.

The original article can be found online at https:// doi.org/10.1007/s40995-021-01199-w.

Nader Jafari Rad

n.jafarirad@gmail.com

Navid Kafai

navid.kafai@kiau.ac.ir

Farideh Heydari

f-heydari@kiau.ac.ir

Mohammad Maghasedi

maghasedi@kiau.ac.ir

1 Department of Mathematics, Karaj Branch, Islamic Azad University, Karaj, Iran

2 Department of Mathematics, Shahed University, Tehran, Iran 